\title{
Acceleration of Magnetospheric Relativistic Electrons by Ultra-Low Frequency Waves: A Comparison between Two Cases Observed by Cluster and LANL Satellites
}

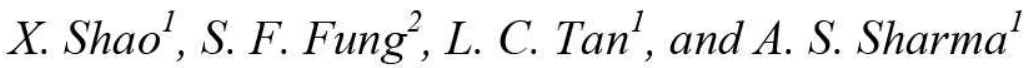 \\ 1. Department of Astronomy, University of Maryland, College Park, MD 20742, USA. \\ 2. Goddard Space Flight Center, NASA, Greenbelt, MD 20771, USA.
}

\begin{abstract}
Understanding the origin and acceleration of magnetospheric relativistic electrons (MREs) in the Earth's radiation belt during geomagnetic storms is an important subject and yet one of outstanding questions in space physics. It has been statistically suggested that during geomagnetic storms, ultra-low-frequency (ULF) Pc-5 wave activities in the magnetosphere are correlated with order of magnitude increase of MRE fluxes in the outer radiation belt. Yet, physical and observational understandings of resonant interactions between ULF waves and MREs remain minimum. In this paper, we show two events during storms on September 25, 2001 and November 25, 2001, the solar wind speeds in both cases were $>500 \mathrm{~km} \mathrm{~s}^{-1}$ while Cluster observations indicate presence of strong ULF waves in the magnetosphere at noon and dusk, respectively, during a 3-hour period. MRE observations by the Los Alamos (LANL) spacecraft show a quadrupling of 1.1-1.5 MeV electron fluxes in the September 25, 2001 event, but only a negligible increase in the November 25, 2001 event. We present a detailed comparison between these two events. Our results suggest that the effectiveness of MRE acceleration during the September 25, 2001 event can be attributed to the compressional wave mode with strong ULF wave activities and the physical origin of MRE acceleration depends more on the distribution of toroidal and poloidal ULF waves in the outer radiation belt.
\end{abstract}

. PACS: Replace this text with PACS numbers; choose from this list: $\underline{\text { http://www.aip.org/pacs/index.html }}$

\section{INTRODUCTION}

Magnetospheric relativistic electrons (MREs) in the outer radiation belt can have hazardous space environment effects on space system. More importantly, during geomagnetic storms/substorms, relativistic electrons can be energized and enhanced in Earth's radiation belt and potentially have serious effects on satellites. Understanding the origin and acceleration of magnetospheric relativistic electrons (MREs) in the Earth's radiation belt during geomagnetic storms is an important subject and yet one of outstanding questions in space physics. Most often, MRE enhancements in the outer radiation belt of the magnetosphere (e.g., geosynchronous region) are correlated with geomagnetic storms and high solar wind speed. But not all storms are associated with MRE enhancements [Reeves 1998]. It was suggested that during geomagnetic storms, increased ultra-lowfrequency (ULF) Pc-5 wave activities in the magnetosphere can enhance the MRE flux by order of magnitude in the outer radiation belt $(\mathrm{L}=5-7)$ and slot region $(\mathrm{L}=2-3.5)$ can be filled with accelerated $\mathrm{MeV}$ electrons [Baker et al., 1998; Rostoker et al., 1998; Green, 2002].

The connection between ULF waves and MRE acceleration has been statistically established by Mathie and Mann [2000], O'Brien et al. [2003]. Statistically [e.g., O'Brien et al. (2001)], significant MRE enhancement tends to occur during: 
- Sustained, high solar wind speed $(>450$ $\mathrm{km} / \mathrm{sec}$ )

- The recovery phase of a magnetic storm

- Long-duration (days) of elevated Pc5 ULF wave power.

In the past, models have been developed to explain MRE acceleration by ULF waves. Schulz and Lanzerotti [1974] developed one dimensional radial diffusion model and related diffusion coefficient with magnetic and electric field wave amplitude. Summers and $M a$ [2000] derived a momentum diffusion model of MRE acceleration by stochastic ULF waves based on quasi-linear theory of wave-particle interaction. Both of these models have neglected the azimuthal distributions of ULF waves. Since electrons drifting in an axially symmetric magnetic field cannot be accelerated by uniformly distributed ULF waves over its drift orbit, asymmetric magnetic field and/or wave distributions are needed for the drift-resonant acceleration mechanism to yield a net energy gain for MREs. For example, Elkington et al. [1999, 2003] considered a compressed dipole field model with daynight asymmetry to investigate the acceleration of magnetospheric electrons by both toroidal and poloidal mode ULF waves. These ULF waves were found to result in electron energization via the drift-resonant interaction.

Degeling et al. [2007] considered the effect of realistic scenario of asymmetric azimuthal distribution of compressional mode ULF waves on electron acceleration and demonstrated that the poloidal mode wave is more efficient in electron energization because of azimuthal overlap of the poloidal ULF electric field and electron drift orbit on the dayside of magnetosphere through modeling. The poloidal mode electric field $\left(\mathrm{E}_{\phi}\right)$ is related to the compressional mode ULF wave that can transfer wave energy across magnetic field lines. The azimuthally asymmetric wave distribution can be attributed to the dominance of wave power on the dayside due to the solar wind compression. Although modeling work indicates that localized toroidal and poloidal ULF waves play different roles on outer belt MRE acceleration, however, there have not been direct observational evidences to distinguish the efficiencies of drift resonant acceleration of MREs between compressional mode and FLR mode ULF waves.

In this paper, we present two events which occurred on September 25, 2001 and November 25, 2001, respectively, to show that although the statistical result supports the general dependences of MREs on solar wind speeds and ULF activities, individual events can behave quite differently. Both events were associated with high solar wind speeds and ( $>3$ hour) strong ULF waves in the magnetosphere. MRE observations by the energetic electron detectors on Los Alamos (LANL) spacecraft showed significant increase $\mathrm{MeV}$ electron flux over a 3-hour period in the September 25, 2001 event, but the MRE increase in the November 25, 2001 event was negligible. We suggest that localized toroidal and poloidal ULF waves play important and distinct roles on outer belt MRE acceleration.

\section{MRE FLUX ENHANCEMENT DURING SEPTEMBER 25, 2001 EVENT}

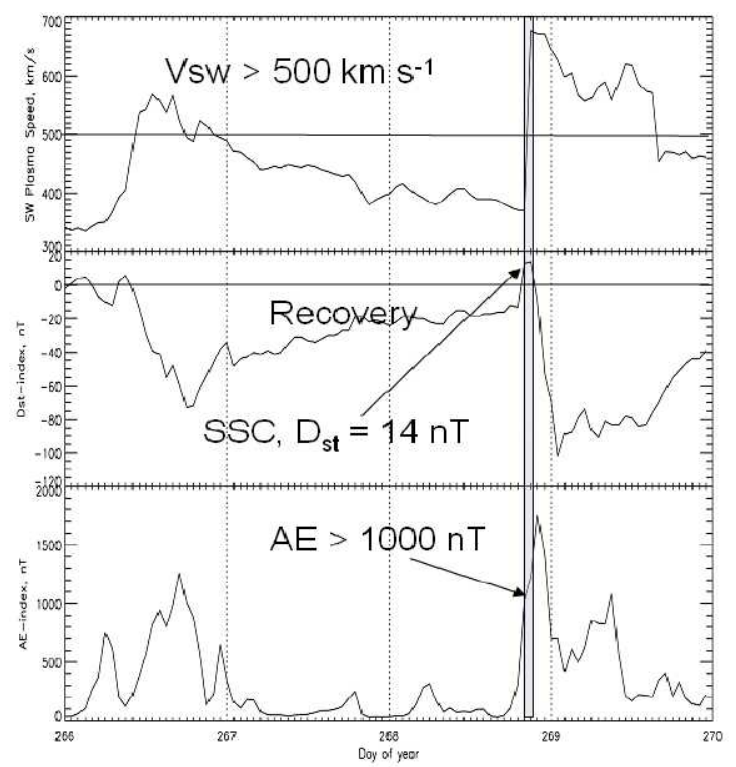

Figure 1: Solar wind speed, Dst index and $\mathrm{AE}$ index during Sept. 23- Sept. 26, 2001. The Sept. 25, 2001 [day of year (doy) 268] storm event is marked with shaded area. (Data source: OMNI).

During the September 25, 2001 event, the MRE enhancement occurred together with significant ULF wave activities. Before the Storm Sudden Commencement (SSC) event occurred on September 25,2001 , there was a geomagnetic storm that occurred on Sept. $23(\mathrm{DOY}=266)$ with Dst minimum $\sim-75$ (Figure 1). It is still in the storm recovery phase during the two days (Sept. 24 and 25) following the Sept. 23 magnetic storm. On September 25, an interplanetary shock driven by the CME arrived at the Earth at 20:25 (UT) and caused a powerful SSC event. The solar wind speed after the shock as measured by the Wind spacecraft was $670 \mathrm{~km} \mathrm{~s}^{-1}$. At the beginning of SSC event the geomagnetic indices were $\mathrm{Kp}=6$ and $\mathrm{AE}=$ $1100 \mathrm{nT}$. During the interval of our interest (UT 20:00 to 23:00 on Sept. 25, 2001), the Dst maximizes around 
$14 \mathrm{nT}$ (signature of SSC), hence the event occurred during the onset phase of the Sept. 25 magnetic storm
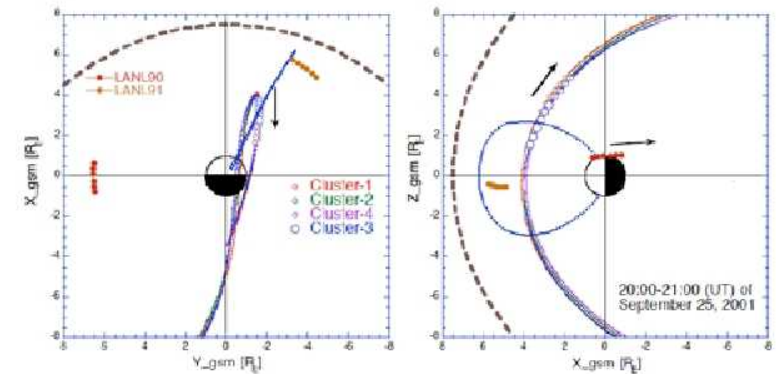

Figure 2: The orbits of the Cluster and LANL90 and 91 spacecrafts at 20:00-21:00 (UT), September 25, 2001. The solid line in the Cluster orbits show the periods when the spacecraft are outside the magnetopause (shown as dashed lines). The dotted blue line (right panel) is a magnetic field line passing through Cluster-3 at 20:30 (UT), computed using the Tsyganenko T89 model.

The SSC event on September 25, 2001 was monitored by Cluster-1 to -4 , and Los Alamos 1990-095 (LANL90), and 1991-080 (LANL91) spacecrafts. In Figure 2, the color-coded open/solid circles show the orbits of the Cluster and LANL spacecrafts during 20:00-21:00 (UT) on September 25, 2001. The arrows near the orbits show the directions of spacecraft motion. The Cluster spacecrafts were in geopolar orbit with perigee near noon and thus were in a suitable location for detecting ULF waves and low-energy electrons near local noon during the September 25 SSC event. Also the orbit of Cluster-3 (largest open circles in Figure 2) was at lower latitudes during the event period. As shown in Figure 2, the magnetic field line (dotted line in Figure 2, right panel) traced using the Tsyganenko T89 model, passed through Cluster-3 at 20:30 (UT), indicating an orbit conjunction between Cluster-3 and LANL91. This conjunction enables comparisons of electron flux measurements between these two spacecraft. The omnidirectional electron flux $\left(J_{\text {omn }}\right)$ data in the electron energy $\left(T_{e}\right)$ range $50 \mathrm{keV}$ $-1.5 \mathrm{MeV}$ were collected by LANL spacecrafts. LANL91 was close to noon, and LANL90 was located at dusk

The Cluster spacecrafts provide the dusk electric field $\left(E_{d u s k}\right)$ data measured in the spin plane perpendicular to the $\mathrm{X}_{\mathrm{GSE}}$ axis and its power spectra from the measurements during 20.20-21.20 hr (UT) of 25 September 2001 as shown in the top panel of Figure 3a. During the event period, $E_{\text {dusk }}$ had a wide-spectral structure. For wave frequency $f_{w}>20 \mathrm{mHz}$, the
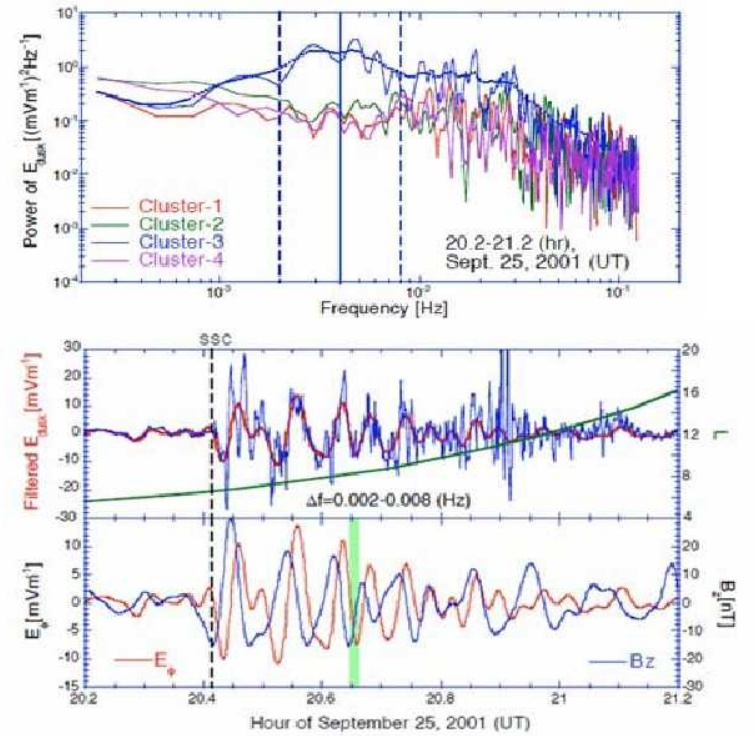

(a)

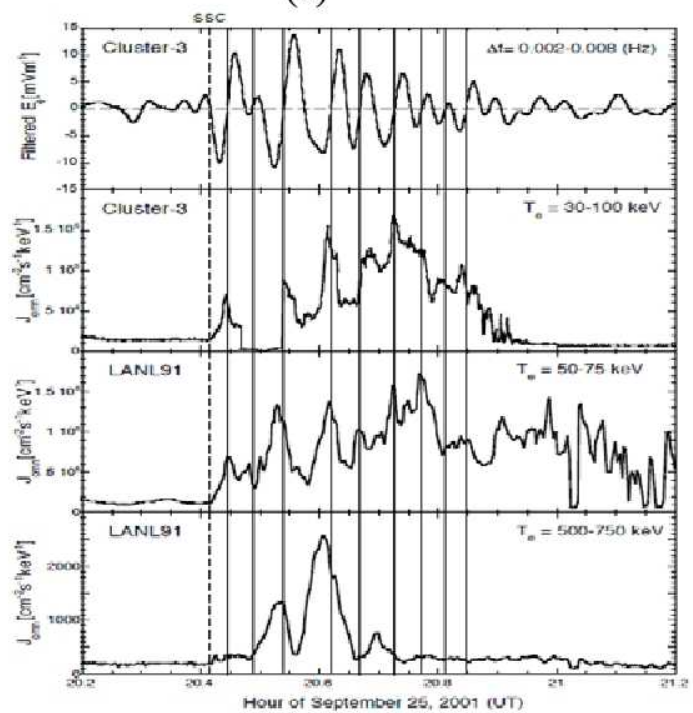

(b)

Figure3: (a) (Top Panel) Power spectra of duskward electric field components from all four Cluster spacecrafts. (Middle Panel) Time profiles of original and filtered $E_{\phi}$, and $L$ values from the Cluster-3 spacecraft observations. (Bottom panel) $B_{z}$ and $E_{\phi}$ after being filtered with same band-pass filter from the Cluster-3 spacecraft observations. (b) Time profiles of filtered $E_{\phi}$ measured by Cluster-3 (top panel), $J_{\text {omn }}$ of $30-100 \mathrm{keV}$ electrons measured by Cluster-3 (second panel), $J_{\text {omn }}$ of $50-75 \mathrm{keV}$ electrons measured by LANL91 (third panel) and $J_{o m n}$ of $500-750 \mathrm{keV}$ electrons measured by LANL91 (bottom panel). The vertical solid lines are the times at which the filtered $E_{\phi}$ crosses zero from negative to positive. 
observed power spectrum of $E_{\text {dusk }}$ by Cluster-1, -2, and -4 are close in magnitude to that on Cluster-3, while at lower frequencies the wave power on Cluster3 is greater than that on other Cluster spacecrafts by more than one order of magnitude. The power of $E_{\text {dusk }}$ measured by Cluster-3 is concentrated in the frequency range of $2-8 \mathrm{mHz}$ (between the dashed lines), which falls right into ULF Pc-5 wave band.

The ULF oscillation of the dusk electric field measured by Cluster- 3 is evident in the filtered data of $E_{\text {dusk }}$ obtained by using a $2-8 \mathrm{mHz}$ band-pass filter. The time profile of filtered electric field is shown in the middle panel of Figure $3 a$ as the red line along with the original electric field data and the $L$ parameter. Since the Cluster-3 spacecraft was located near noon during the September SSC event, $E_{\text {dusk }}$ can be viewed as the azimuthal electric field component and we denote it as $E_{\phi}$ in the rest of the paper.

In bottom panel of Figure 3a, we compare the phase of $E_{\phi}$ with that of $B_{z}$, which is filtered using the same band-pass filter. It can be seen that over the main observation interval between SSC and $20.65 \mathrm{hr}$ (UT) (the shaded green region), $B_{z}$ led $E_{\phi}$ by $\sim \pi / 2$, while after $20.65 \mathrm{hr}$ (UT) both phases are gradually close to each other. In addition, the magnetic field amplitude observed by GOES satellite has ULF oscillations [Tan et al., 2010]. These features are consistent with the simulations of compressional mode waves [Degeling et al., 2007, Figure 1]. These waves are poloidal mode waves and are related to radial perturbation of magnetic field line. It is of fast-mode wave nature and is related to the perturbed field components $\delta B_{\rho}, \delta E_{\phi}$ and $\delta B_{z}$ [Takahashi et al., 1992, 1995].

The modulation of low-energy electron fluxes by the compressional mode ULF waves can be identified using the wave data from Cluster and electron data from Cluster and LANL spacecrafts. In Figure $3 b$ the time profile of filtered $E_{\phi}$ oscillations (top panel) is compared with $J_{\text {omn }}$ for Cluster-3 30-100 keV electrons (see middle panel of Figure $3 \mathrm{~b}$, the differential flux is deduced from Cluster-3 integrated flux data), and for LANL91 50-75 keV electrons (see bottom panel of Figure 3b). Because of the conjunction between Cluster-3 and LANL91 orbits, the phases of $J_{\text {omn }}$ measured on both spacecraft matched with each other very well. On the other hand, there is no clear modulation of higher energy (500-750
$\mathrm{keV}$ ) electron flux as observed by LANL 91 (see bottom panel of Figure 3b).

The acceleration of relativistic electrons can be identified from the omnidirectional electron flux $J_{\text {omn }}$ measured by GOES 08-10 and LANL 90 shown in Figure $4 \mathrm{a}$. Figure $4 \mathrm{~b}$ shows the $J_{\text {omn }}$ data over a wide energy range ( $50 \mathrm{keV}-1.5 \mathrm{MeV})$ as measured by the LANL90 spacecraft located at dusk. It can be seen that low-energy electrons did not have net energy increase, since the resultant level of $J_{\text {omn }}$ for LANL90 is nearly equal to the pre-event background value after 21:40 UT at the end of modulation.

After the anomalous increase of $J_{\text {omn }}$ during the first hour after the SSC onset, a steady diffusive enhancement of high-energy (e.g., 1.1-1.5 MeV) electron flux was observed $\sim 1.5$ hours after the SSC onset. With respect to the pre-event backgound value the electron flux $J_{\text {omn }}\left(T_{e}=1.1\right.$ $1.5 \mathrm{MeV}$ ) enhancements observed by LANL90 is 4 times. There is a threshold $T_{e}(t h r)$ of electron energy, below which the electron fluxes are highly modulated by the ULF waves and the final electron flux doesn't exceed pre-event background value. Above this threshold the electron flux increases diffusively and the final electron flux has a net increase. The electron energy thresholds $\left(T_{e}(t h r) \sim 300 \mathrm{keV}\right)$ as identified from LANL90 observations are shown as the horizontal purple lines in Figure $4 \mathrm{~b}$. The implication of this energy threshold and theory of drift-resonant acceleration of MREs by compressional mode ULF waves can be found in Tan et al. [2010].

\section{NOVEMBER 25, 2001 EVENT WITH STRONG ULF WAVE ACTIVITY, BUT NO NET MRE ACCELERATIONS}

The November 25, 2001 event occurred during the recovery phase of a large geomagnetic storm as shown by the Dst index in Figure $6 \mathrm{~b}$ and the associated interval of extremely long-lasting ULF Pc5 pulsations has been analyzed extensively by Rae et al. [2005]. Rae et al. [2005] reported that these monochromatic large-amplitude ULF 


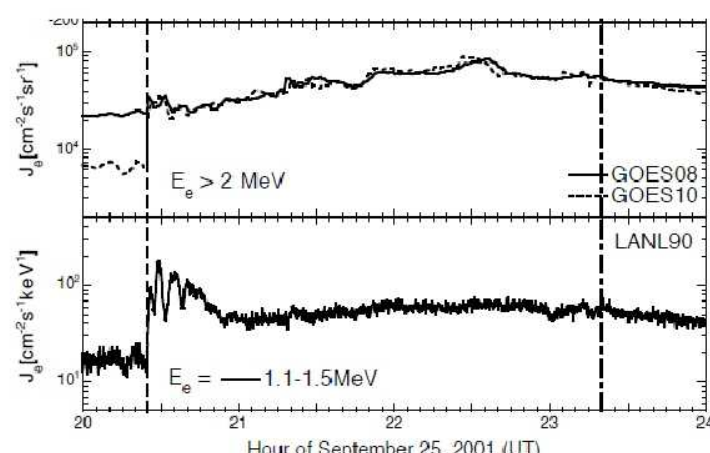

(a)

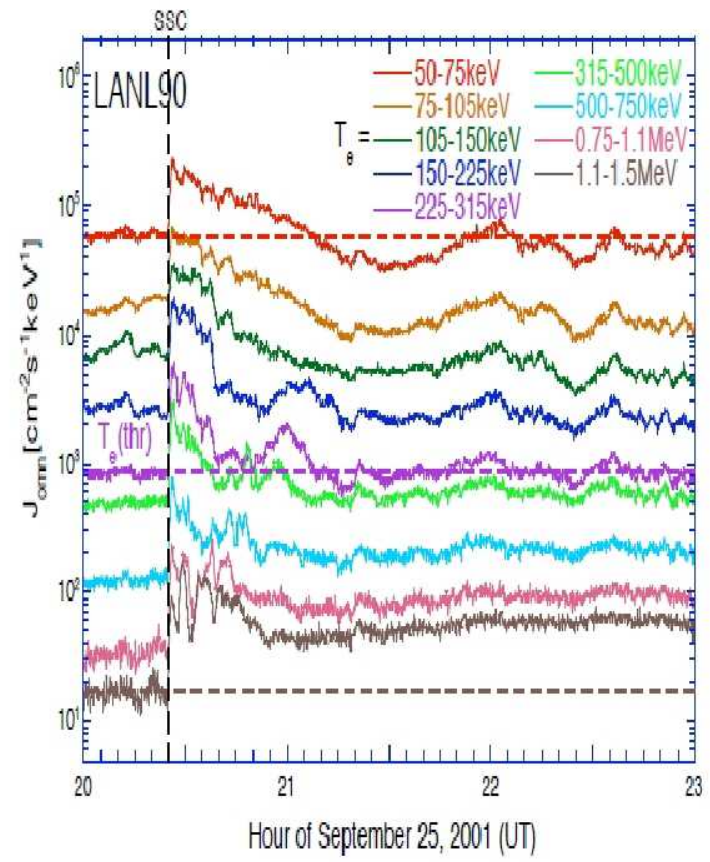

(b)

Figure 4: (a) (Top panel) GOES08 and GOES10 satellite observation of $>2 \mathrm{MeV}$ MRE flux enhancement. (Bottom panel) LANL90 satellite observation of 1.1-1.5 MeV MRE flux enhancement. (b) Time profiles of $J_{o m n}$ measured by LANL90. The upper, middle and lower horizontal dashed lines indicate the background $J_{\text {omn }}$ levels at $T_{e}=50-75$ $\mathrm{keV}, T_{e}=225-315 \mathrm{keV}$ and $T_{e}=1.1-1.5 \mathrm{MeV}$, respectively.

pulsations occurred continuously for many hours and were observed throughout the

magnetosphere by the Cluster satellites, and geostationary satellites GOES-8, $-9,-10$ and -12 , and in the dusk-sector ionosphere by the CANOPUS magnetometer chain. The Cluster satellites are in the vicinity of the dusk magnetopause (Figure 7) and show oscillations with the same periodicity as the ground and geosynchronous pulsations. As an example, the ULF oscillation can be seen from Figure $6 \mathrm{~d}$ which shows the bandpass-filtered duskward electric field observed by Cluster- 4 during the interval. In Figure 8a, low energy electron flux shows ULF modulated oscillation and no net increase.

During this event, there are no monochromatic dynamic pressure variations in the solar wind. It is suggested by Rae et al. [2005] that Kelvin-Helmoltz instability at the duskside magnetopause caused magnetopause oscillations, which coupled energy to standing Alfvén waves propagating along the resonant field lines. This explains that ground-based magnetometers observed signatures characteristic of a field-line resonance. The standing Alfven wave is of toroidal mode wave nature and is related to azimuthal perturbation of magnetic field line. It propagates along the magnetic field line and is related to the perturbed field components $\delta B_{\phi}$ and $\delta E_{r}$.

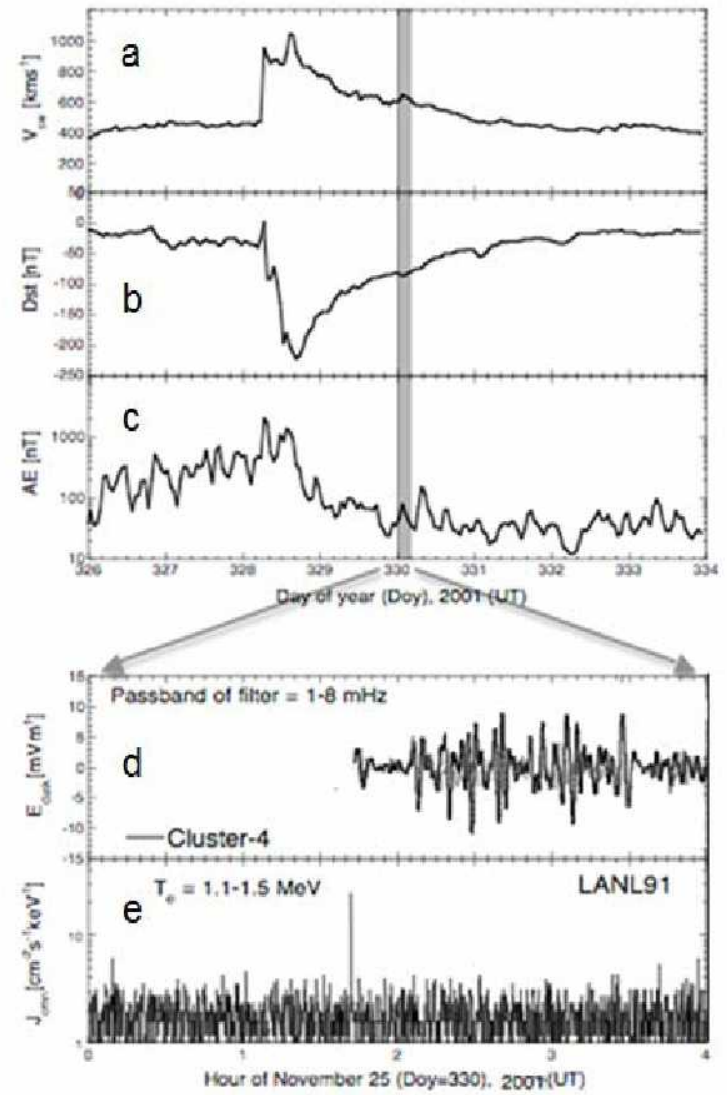

Figure 6: (a-c) Solar wind speed, Dst index and $\mathrm{AE}$ index during Nov. 21- Nov. 28, 2001. (Data source: OMNI) (d) Strong ULF wave activities observed by Cluster-4 spacecraft. Earlier data before UT 1:40 was a data gap. (e) No MRE flux enhancement observed by LANL 91. 
The evidence of toroidal mode wave can be also seen in Figure $8 b$, which shows that the radial electric perturbation carried the largest energy spectra as observed by the Cluster-2 spacecraft in the vicinity of duskside magnetopause.
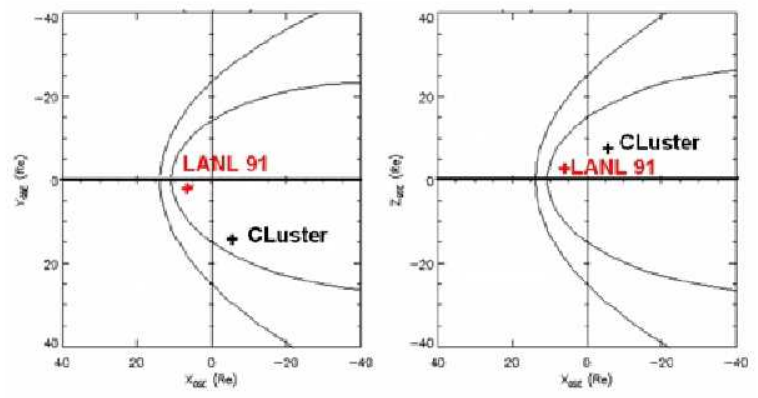

Figure 7: Cluster and LANL 91 spacecraft location during the November 25, 2001 event.

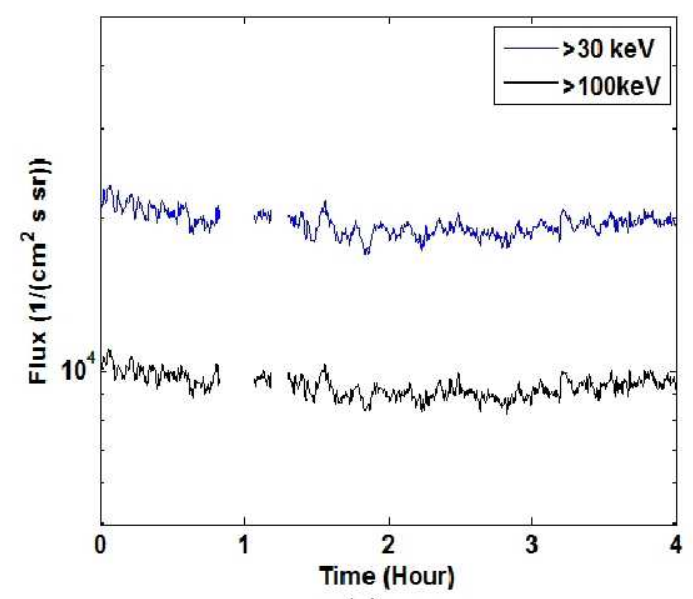

(a)

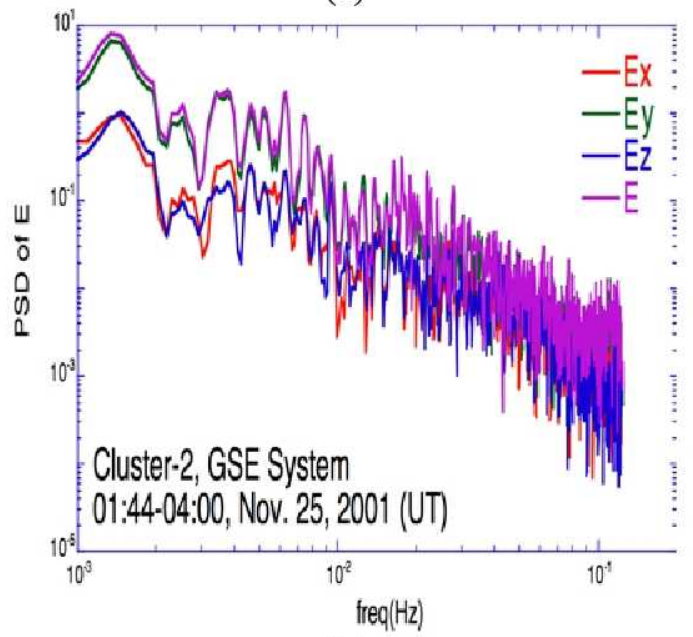

(b)

Figure 8: (a) electron flux observed by Cluster-4 spacecraft. (b)Power spectra of electric field components for Cluster-2 spacecraft. Since the Cluster satellite is located at the dusk side, the dominant electric field component $E_{y}$ can regarded as $E_{r}$.

During this event interval, the solar wind velocity $\mathrm{V}_{\mathrm{sw}}$ $>500 \mathrm{~km} / \mathrm{s}$, and it is during the recovery phase of a large storm with minimum Dst $<-200 \mathrm{nT}$. Also, strong Pc5 ULF activity near $1.5 \mathrm{mHz}$ were observed throughout the magnetosphere and duskside ionosphere. All of these factors meet the criteria derived from the statistical analysis by O'Brien et al. [2001], which implies that these conditions will favor MRE acceleration. But, there is no net MRE enhancement observed, i.e. for electron of energy $T_{e}=$ 1.1-1.5MeV by LANL 91 observation in Figure 6e. Instead, Rae et al. [2005] reported coherent low energy (75-315 keV) electron flux modulations by ULF waves from observations of LANL spacecrafts distributed at different local time around the geosynchronous orbit.

\section{COMPARISON BETWEEN SEPTEMBER 25, 2001 AND NOVEMBER 25, 2001 EVENTS}

In this section, we do a comparison between September 25, 2001 and November 25, 2001 events and investigate factors affecting efficient MRE accelerations by ULF waves.

Table 1 compares features of the two events we studied. In general, the solar wind velocities are high ( $>500 \mathrm{~km} / \mathrm{s}$ ). Also, strong ULF fluctuations are both observed. But, the September 25, 2001 event observed MRE enhancement and the November 25, 2001 event did not. During the first 1.5 hours after SSC onset in September 25, 2001 event, the low-energy electron flux observed by LANL 90 exhibits less modulation as compared to the modulation observed in Figure $3 \mathrm{~b}$ by LANL 91 on the dayside. This indicates that the ULF wave energy is mainly concentrated in the dawn-noon sector of the dayside where LANL-91 is located and less disturbed at the dusk side where LANL 90 is located. This further suggests that the ULF wave energy is fed through dayside compression rather than Kelvin-Helmholtz instability or other processes at the flanks.

We found that the main difference between these two events are that during the SSC

event of September 25, 2001, the ULF waves are mainly poloidal mode waves and concentrated at and near the outer radiation belt in the dayside magnetosphere; and during the event of November 25 , 2001, Kelvin-Helmholtz instabilities due to magnetosheath shear flows excited flank side- 
concentrated field line resonance (toroidal mode waves). These findings are consistent with previous observations. For example, Engebretson et al. [1998] and Vennerstrom [1999] observed that Pc-5 wave power tends to maximize near magnetic local noon and increases more generally with solar wind velocity such

\begin{tabular}{|c|c|c|}
\hline Date & $\begin{array}{l}\text { September } 25 \\
00-04 \text { UT }\end{array}$ & $\begin{array}{l}\text { November } 25 \\
20-24 \text { UT }\end{array}$ \\
\hline $\begin{array}{l}\text { MRE ( 1 MeV) } \\
\text { enhancement }\end{array}$ & Yes & No \\
\hline Vsw & $\begin{array}{l}500 \mathrm{~km} \mathrm{~s}-1 \\
\text { (implusive) }\end{array}$ & $>500 \mathrm{~km} \mathrm{~s}-1$ \\
\hline Enhanced ULF & Yes & Yes \\
\hline Storm condition & Onset & Recovery \\
\hline $\mathrm{SSC}$ & Yes & No \\
\hline $\mathrm{Bz}$ (GSE) & Mostly $<0$ & Mostly $>0$ \\
\hline Solar wind Pdyn & $>20 \mathrm{nPa}$ & $-2 \mathrm{nPa}$ \\
\hline ULF characteristics & $\begin{array}{l}\text { Stronger E } \phi \text {, } \\
\text { Poloidal }\end{array}$ & $\begin{array}{l}\text { E } \rho \text {, Weaker E } \phi \text {, } \\
\text { Toroidal }\end{array}$ \\
\hline $\mathrm{AE}$ & $>1000 \mathrm{nT}$ & $<100 \mathrm{nT}$ \\
\hline
\end{tabular}

Table 1: Comparison between the two events.

as during high-speed streams. Sakurai et al. [1999] also found that compressional power is superior to that of the transverse resonant oscillations when spacecraft was close to the magnetopause. These oscillations during SSC events have been suggested to be excited by waves or compression regions in the solar wind [Kepko and Spence, 2003; Kessel et al. 2003; Kessel and Shao, 2005]. On the other hand, Anderson et al. [1990] observed that storm-time Pc-5 waves (fundamental mode of Field Line Resonance (FLR)) are excited primarily in the dusk and dawn regions and by magnetosheath flows [Mann et al. 1999, 2004].

\section{EFFICIENCIES OF DRIFT-RESONANT ACCELERATION BY POLOIDAL AND TOROIDAL ULF WAVES}

The electron acceleration by poloidal mode wave $\left(E_{\phi}\right)$ should be more effective than by toroidal mode wave $\left(\mathrm{E}_{\mathrm{r}}\right)$. This is because that $\mathrm{E}_{\mathrm{r}}$ is perpendicular to the electron drift orbit and its energization effect depends on the drift orbit deviation
$\Delta \mathrm{r}$ due to the magnetospheric compression. Since the main component of electron drift motion is along the azimuthal direction, interaction of electrons with compressional mode waves whose electric field is along the azimuthal direction, can be more effective in generating MREs. We can use a compressed dipole magnetic field to approximate the equatorial magnetic field configuration [Elkinton et al., 2003] and illustrate on this. In the model the magnetic field strength $B$ is a function of the radius $\mathrm{r}$ and azimuthal angle $\phi$,

$B(r, \phi)=b_{0}+b_{1}\left(1+b_{2} \mathrm{c} \phi\right)$,

where $b_{0}=B_{0} R_{E}^{3} / r^{3}\left(\mathrm{~B}_{0}=3.11 \times 10^{4} \mathrm{nT}\right), \mathrm{b}_{1}$ and $\mathrm{b}_{2}$ are model parameters. Alternatively, we can use two new parameters $B_{\mathrm{m}}=b_{0}+b_{1}\left(1+b_{2}\right)$ and $B_{\mathrm{m}}=b_{0}+b_{\mathrm{h}}\left(1-b_{2}\right)$ to describe the model, which correspond to day-night asymmetry in magnetic field configuration [Tan et al., 2004]. Thus the relative acceleration efficiency of toroidal mode to poloidal mode wave $\left(E_{r}\right.$ to $\left.E_{\phi}\right)$ can be related to the factor $\Delta r / r$ using the compressed dipole model,

$\Delta r / r \sim\left(b_{\max }-b_{\min }\right) L^{3} /\left(6 B_{0}\right)$.

During September 25, 2001 event, we determined $B_{\min }$ $\sim 65 \mathrm{nT}$ and $\mathrm{B}_{\max } \sim 190 \mathrm{nT}$ from the magnetic field $\mathrm{B}_{\mathrm{z}}$ component measured by GOES10. Relative acceleration efficiency of $E_{r}$ to $E_{\phi}$ (Eq. 2) has a value of $\sim 0.15$ at $\mathrm{L}=6$. Therefore, in the September 25 event the electron acceleration is mainly due to the poloidal mode electric field $\mathrm{E}_{\phi}$. This also explains that the observed lack of MRE enhancement in the November 25, 2001 event is consistent with less effective acceleration by toroidal mode waves.

\section{SUMMARY}

In this paper, we compared two magnetic storm events, one with and one without MRE enhancements. We found that either toroidal or poloidal mode can both produce electron flux modulations for low energy electrons. It is suggested that during a sudden storm commencent (SSC), i.e. 2001 September 25. SSC, events tend to produce dayside compressive mode with $\mathrm{E}_{\phi} \quad$ perturbations, producing $(\mathrm{MeV})$ MRE enhancement. On the other hand, radial electric field oscillations E $\rho$ (e.g., toroidal field line resonances) on the flanks are not effective in producing MREs, as was observed in the 2001 November 25 event. In Tan et al. [2010], more detailed theory and analysis on the drift-resonant acceleration of MREs by compressional mode wave as inferred from September 25, 2001 event 
will be discussed. A more comprehensive study of the relationships among upstream solar wind and IMF conditions, Ultra-Low-Frequency (ULF) waves, and Magnetospheric Relativistic Electrons (MREs) is needed, in particular, to study the excitation and distribution of ULF waves by upstream solar wind and IMF conditions and the drift resonant acceleration of relativistic electrons by ULF waves.

\section{ACKNOWLEDGEMENTS}

This work is dedicated to celebrate the career of Prof. Dennis Papadopoulos' career. X. Shao's work is supported partially by NASA grant and NSF grant. $X$. Shao and A. S. Sharma's work is supported by ONR MURI grant.

\section{References:}

Anderson, B.J., M.J. Engebretson, and S.P. Rounds, A statistical study of Pc 3-5 pulsations observed by the AMPTE/CCE magnetic fields experiment 1. Occurrence distributions, J. Geophys. Res., 95, A7, 10,495-10,523, 1990.

Baker, D. N., T. I. Pulkkinen, X. Li, S. G. Kannel, J. B. Blake, R. S. Selesnick, M. G. Henderson, G. D. Reeves, H. D. Spence, and G. Rostoker, Coronal mass ejections, magnetic clouds, and relativistic magnetospheric electron events: ISTP, J. Geophys. Res., 103, 17279, 1998a.

Baker, D. N., T. I. Pulkkinen, X. Li, S. G. Kannel, K. W. Ogilvie, R. P. Lepping, J. B. Blake, L. B. Callis, G. Rostoker, H. J. Singer, G. D. Reeves, A strong CMErelated magnetic cloud interaction with the Earth's magnetosphere: ISTP observations of rapid relativistic electron flux acceleration on May 15, 1997, Geophys. Res. Lett., 25, 2975, 1998b.

Degeling, A. W., et al., 2007, The effect of ULF compressional modes and field line resonances on relativistic electron dynamics, Planet. Space Sci., 55, 731.

Elkington, S. R., et al., 1999, Acceleration of relativistic electrons vis drift-resonant interaction with toroidalmode Pc-5 ULF oscillations, Geophys. Res. Lett., 26, 3273.

Elkington, S. R., et al., 2003, Resonant acceleration and diffusion of outer zone electrons in an asymmetric geomagnetic field, J. Geophys. Res., 108(A3), 1116, doi:10.1029/2001JA009202.

Engebretson, M., K.-H. Glassmeier, M. Stellmacher, W.J. Hughes, H. Luehr, The dependence of high- latitude Pc5 wave power on solar wind velocity and on the phase of high-speed solar wind streams, J. Geophys. Res., 103, A11, 26,271-26,283, 1998.

Green, J.C., Testing Relativistic Electron Acceleration Mechanisms, Ph.D. thesis, UCLA, 2002.
Kepko, L. and H. E. Spence, Observations of discrete, global magnetospheric oscillations driven by solar wind density variations, J. Geophys. Res., 108(A6), 1257, doi:10.1029/2002JA009676, 2003.

Kessel, R. L., I. R. Mann, S. F. Fung, D. K. Milling, and N. O'Connell, Correlation of Pc5 wave power inside and outside the magnetosphere during high speed streams, accepted by Annales Geophysicae, 2003.

Kessel, M., and X. Shao, How does the solar wind power the magnetosphere during Geo-effective high speed streams, Multiscale coupling of Sun-Earth Processes, Edited by A.T.Y. Lui, Y. Kamide, and G. Consolini, Elsevier B. V., 2005.

Mann, I. R., A. N. Wright, K. J. Mills, and V. M. Nakariakov, Excitation of magnetspheric waveguide modes by magnetosheath flows, J. Geophys. Res., 104, 333, 1999.

Mann, I. R., T. P. O'Brien, D. K. Milling, Correlations between ULF wave power, solar wind, and relativistic electron flux in the magnetosphere: solar cycle dependence, J. Atmos. Sol-Terres. Phys., 66, 187, 2004.

Mathie, R.A., and I.R. Mann, A correlation between extended intervals of ULF wave power and storm-time geosynchronous relativistic electron flux enhancements, Geophys. Res. Lett., 27, 3261-3264, 2000.

Nakamura, M., H. Matsu, H. Kawano, S. Kokubun, K. Takahashi, A. Matsuoka, T. Yamamoto, K. Tsuruda, and H. Hayakawa, Pc5 pulsations observed in the dayside magnetosphere by Geotail, Geophys. Res. Lett., 21, 2903, 1994

O'Brien, T. P., R. L. McPherron, D. Sornette, G. D. Reeves, R. Friedel, and H. J. Singer (2001), Which magnetic storms produce relativistic electrons at geosynchronous orbit?, J. Geophys. Res., 106(A8), 15,533-15,544.

O'Brien, T.P. K.R. Lorentzen, I.R. Mann, N.P. Meredith, J.B. Blake, J.F. Fennell, M.D. Looper, D.K. Milling, and R.R. Anderson, Energization of relativistic electrons in the presence of ULF power and $\mathrm{MeV}$ microbursts: evidence for dual ULF and VLF acceleration, J. Geophys. Res., 108 (A8), 1329, doi:10.1029/2002JA009784, 2003.

Rae, I. J., E. F. Donovan, I. R. Mann, F. R. Fenrich, C. E. J. Watt, D. K. Milling, M. Lester, B. Lavraud, J. A. Wild, H. J. Singer, H. Rème, and A. Balogh (2005), Evolution and characteristics of global Pc5 ULF waves during a high solar wind speed interval, J. Geophys. Res., 110, A12211, doi:10.1029/2005JA011007.

Reeves, G. D. (1998), Relativistic electrons and magnetic storms: 1992-1995, Geophys. Res. Lett., 25(11), $1817-$ 1820.

Rostoker, G., S. Skone, and D.N. Baker, On the origin of relativistic electrons in the magnetosphere, Geophys. Res. Lett., 25, 3701-3704, 1998.

Sakurai, T., et al., Dayside magnetopause Pc 3 and Pc 5 ULF waves observed by Geotail satellite, Earth Planet. Space, 51, 965, 1999.

Schultz, M., and L. J. Lanzerotti, Physics and Chemistry in Space, vol. 7, Particle Diffusion in the Radiation Belt, Springer-Verlag, New York, 1974.

Summers, D., Ma, C., Rapid acceleration of electrons in the magnetosphere by fast-mode MHD waves, J. Geophys. Res., 105, 887, 2000 
Takahashi, K., and B.J. Anderson, Distribution of ULF energy $(\mathrm{f}<80 \mathrm{mHz})$ in the inner magnetosphere - A statistical analysis of AMPTE CCE magnetic-field data, J. Geophys. Res., 97, A7, 10751-10773, 1992.

Takahashi, K., Shin-ichi Ohtani, and B. J.Anderson, Statistical analysis of $\mathrm{Pi} 2$ plsations observed by the AMPTE CCE spacecraft in the inner magnetosphere, $J$. Geophys. Res., 100, 21,929, 1995.

Tan, L. C., S. F. Fung, and X. Shao, Observation of magnetospheric relativistic electrons accelerated by Pc5 ULF waves, Geophys. Rev. Lett., L14802, doi: 10.1029/2004GL019459, 2004.

Tan, L. C., S. F. Fung, X. Shao, and A. S. Sharma, (2010), Relativistic Electron Acceleration by Compressional Mode ULF Waves: Evidence from Correlated Cluster, LANL Spacecraft and Ground-Based Magnetometer Measurements, to be submitted to J. Geophys. Res.

Vennerstrom, S., Dayside magnetic ULF power at high latitudes: A possible long-term proxy for the solar wind velocity?, J. Geophys. Res. 104, A5, 10,145-10,157, 1999. 